\title{
Telemetry in Disaggregated Optical Networks
}

\author{
Francesco Paolucci, Andrea Sgambelluri \\ CNIT PNT Lab - Scuola Superiore Sant'Anna \\ Pisa, Italy \\ fr.paolucci@santannapisa.it
}

\begin{abstract}
Advanced monitoring plane enabling on-demand real-time transmission parameters detection and analysis will drastically improve seamless control and management of future optical networks. The tutorial reports the concept, along with the state-of-the-art and proposed design and implementations related to telemetry-augmented Elastic Optical Networks and next-generation metro networks. Focus will be given to SDNcontrolled networks exploiting disaggregation and discussion on current telemetry models in the OpenROADM and OpenConfig open source initivatives targeting multi-vendor interoperability.

Index Terms-White box, gRPC, Thrift, IPFix, OpenConfig, OpenROADM, streaming, optical monitoring.
\end{abstract}

\section{INTRODUCTION}

Elastic Optical Networks (EON) are evolving in the last years towards improved flexibility, multi-domain and multivendor interoperability thanks to the disaggregation paradigm, and advanced AI-driven monitoring functions.

The detailed and combined monitoring of optical connections behavior, along with the physical parameters monitoring disclosed by optical devices (e.g., coherent receivers) are considered essential nowadays to increase network awareness, to identify soft failures, to detect correlation between anomalous events, to localize and to predict/forecast malfunctioning devices before their actual fault [1]. For example, the study of the received spectrum at Optical Spectrum Analyzer (OSA) placed within specific devices and correlations among such spectra may reveal WSS filter malfunctioning (e.g, filter shift, tightening) [2]. On the other hand, soft failures affecting amplifiers may be extremely hard to detect when automatic gain control is employed [3].

The availability of large monitoring datasets is becoming feasible thanks to massive cloud and edge computational and storage resources, along with synchronized distributed database technologies. Standard monitoring procedures (e.g., 15-minutes statistics) and management protocols (e.g. SNMP) are not able to provide effective time data plane monitoring in terms of detailed both end-to end and per-node signal power and quality stability, device malfunctioning and equipment/fiber ageing. Real time monitoring has been proved to provide efficient network operation and a reduction of the total cost of ownership in the long term [4].

Telemetry relies on the autonomic network concept and is defined as the collection of online samples of monitored data

This project has received funding from the ECSEL Joint Undertaking (JU) under grant agreement No 876967. The JU receives support from the European Union's H2020 research and innovation programme and from Italy Ministry of Education, University and Research (MIUR). from many optical devices provided by a dedicated service, typically relying on a streaming connection. The service specifies the data sample type to be retrieved. Examples of significant parameters to be sampled at the EON transceiver coherent receiver are: the Bit Error Rate (BER), the Optical Signal to Noise Ratio (OSNR), input/output power. Additional parameters are the monitored optical spectrum power level, the actual amplification gain and tilt.

Besides major faults (e.g., fiber cut, node down), telemetry services combined with efficient AI-based processing tools are able to detect additional critical events such as soft failures (e.g., sporadic malfunctioning), QoT degradations due to fiber mechanical or temperature solicitations, optical filter deviations, ageing. Minor events may be detected or predicted and localized, thus allowing improved network maintenance and reducing the probability of possible major failure events.

\section{TELEMETRY SERVICE ARCHITECTURES IN DISAGGREGATED NETWORKS}

The optical network disaggregation, combining the white box concept and SDN standard API, allows flexible configurability and monitoring of multi-vendor node components. Depending on the disaggregation degree, telemetry service may be enabled and triggered on-demand over a node or a single white box device.

The typical telemetry service architecture includes both control plane and monitoring/management plane modules and is shown in Fig. 1, along with the typical activation workflow. Control plane southbound API (e.g., YANG/NETCONF), besides standard configuration set and get functions (step 1), includes procedures to request the activation of a given dataset telemetry service to be retrieved from a set of SDN agents. Activation may be either manually triggered by the operator maintenance task force or automatically started by the control/management plane in the case specific notification events are received (step 2). The agent may be a node controller (e.g., ROADM controller) or a single device controller (e.g., the degree module inside a ROADM, or a Xponder card). Typically, telemetry activation relies on subscription of the requested dataset, along with its attributes and parameters, over the target agent (step3). The agent runs a dedicated telemetry server, in charge of collecting monitored data samples from the underlying hardware card driver. After subscription, telemetry data are then transmitted under the form of standard data connection or resorting to streaming connection (step 4). Data are received by a collector (i.e., a database location) for 
data storage and analytics purposes. The module in charge of processing and interpreting telemetry, referred to as Operation Administration Maintenance (OAM) Handler, instructs control/management plane how to react properly (step 5).

Typically, two alternative subscription workflows are implemented: the dial-in and the dial-out workflows. In the former, the controller triggers the collector to connect to the device agent. The collector then subscribes to the desired parameters and receives the data streaming. Instead, in the latter, the controller configures the device agent specifying parameters, telemetry attributes and collector addresses. Then, the device agent is in charge of connecting to the selected collector and streaming data. The disaggregated telemetry service shown in Fig.1 implements a dial-out workflow.

\section{A. Fully disaggregated telemetry}

Disaggregation allows to extend telemetry workflows and to improve service scalability. In fact, in the case of full disaggregation (e.g., Xponder cards and optical line systems made of white boxes), telemetry may take place between domain controller and SDN agents, but also between node controller (i.e., white box controller) and related node device agents. In the case the node controller has computational and storage capabilities (e.g., edge node co-located), telemetry service may be offloaded, splitted and confined inside each node by a dedicated Node Handler, thus providing processed telemetry results directly to the domain controller. Thus, full disaggregation enables a hierarchical telemetry system, in which an upper layer element may offload all the telemetry service at the node level. This implies improved scalability at controllers, at the cost of an increased number of interactions and computational resources inside the single network node.

\section{B. Partially disaggregated telemetry}

In the case of partial disaggregation (e.g., vendor independent Xponders mounted over a single-vendor optical line system) or in presence of legacy equipment (i.e., black box), the telemetry can not be offloaded and splitting is not complete. In this case the domain controller is in charge to coordinate telemetry services. To guarantee scalability (i.e., to allow parallel telemetry streaming of a potentially massive amount of device physical parameters), subscriptions may require a set of parallel monitored data over the same streaming packet, called data bundling [3]. Moreover, some telemetry protocols allow compressed data transmission (e.g., by utilizing implicit header and field formats), thus further reducing the telemetry streaming bandwidth rate. This implies an increased complexity at the telemetry agent to handle parallel and optimized streaming sessions.

\section{SDN TELEMETRY YANG MODELS AND PROTOCOLS}

The main considered YANG models supporting SDN disaggregated telemetry services are developed by OpenConfig, lead by Google, and OpenROADM lead by AT\&T.

The OpenConfig YANG model, designed to support mainly Xponders, include a dedicated telemetry configuration tree.

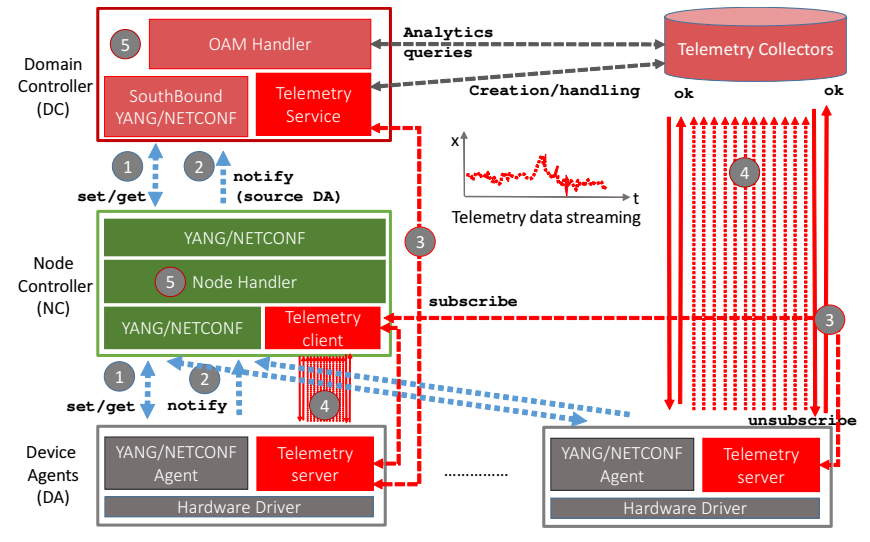

Fig. 1. Telemetry service architectures and workflow

The resource set to be monitored is defined by the sensor group, including a list of sensor-path tags that identifies the path in the general model pointing to target fields belonging to the sensor group. The local source and the destination group specify the source and destination IP collectors, respectively. Subscriptions are of type persistent, configured locally on the device and persistent against restart or reboot, or dynamic, triggered through RPC channel and not persisting after restart or RPC reset. Additional configurable parameters are the heartbeat-config (i.e., the stream packet update rate), the protocol (XML, JSON, Proto3 are supported), the data encoding (SSH, JSON-RPC, Thrift, WebSocket and gRPC are supported), destination ports, and the stream-frequency-config (i.e., data collection time granularity, if set to 0 the generation rate is event-based).

The OpenROADM initiative, promoted by a consortium of network operators, defines a complex and detailed YANG model for each node device and component, targeting full disaggregation. Recently, OpenROADM has inherited and incorporated the OpenConfig telemetry model branch inside its model, substantially supporting the same options over a wider set of device parameters disclosed by the model itself.

\section{A. Telemetry protocols and collectors}

The most utilized telemetry protocols in real implementations are IPFix [5], Thrift [6], and gRPC [3]. IPFix is a push protocol defined by IETF to perform metering and data collection process from observation points towards collectors, without any additional interaction by the receiver. Thrift and gRPC both provide interface definition languages and communication protocols. The main difference between Thrift and gRPC is that gRPC natively supports asynchronous-triggered and bidirectional streaming, particularly useful for telemetry services. gNMI is a promising Google-driven gRPC management protocol providing full configuration of network devices, including telemetry service. Data received by collectors are handled, processed and synchronized by means of big data analytics frameworks (e.g, Hadoop, Apache Spark, Apache Kafka) and AI platforms relying on standard libraries (e.g., TensorFlow, PyTorch and Keras API). 


\section{STATE-OF-THE-ART AND IMPLEMENTATIONS}

The work in [7] is one of the first studies proposing YANG extensions for on-demand data acquisition from optical device agents, resorting to IETF Abstraction and Control of TE Networks (ACTN) virtual network models. The work [8] proposes the use of data analytics including IPFix observation points to sliced optical network based on the Spark platform. The work has been extended in [5] to support white boxes, perform OSA spectrum telemetry and detect/recover optical filter malfunctioning thanks to the combined use of ONOS controller and CASTOR Monitoring and Data Analytics tool. Multi-layer extensions have been covered in [9]. The work in [3] presents two main architectural implementations of telemetry based on partial and full disaggregation degrees, using gRPC. Authors declare $200 \mathrm{~kb} / \mathrm{s}$ for 100 subscribed devices using 1s sampling time and a gRPC encoding 4 times more efficient than NETCONF XML.

The work in [10] proposes a disaster recovery use case employing a highly reconfigurable telemetry system to flexibly react to post-disaster low bandwidth and unstable data and control network conditions. The work in [11] proposes an online gRPC telemetry of physical fiber parameters (i.e., bending) affecting coherent receiver OSNR, combined with AI-based convolutional network. The work [12] proposes a power monitor blade with ultra high telemetry streaming rate and control plane using direct access memory to support fast AI. Authors declare a telemetry update of 400 us. The work [13] shows an open disaggregated ROADM including filterless add/drop module equipped with photodetector tap arrays with gRPC telemetry servers including NETCONF notifications. Authors declare $20 \mathrm{~Hz}$ of sample update frequency. Work in [14] proposes an open source OpenConfig SDN agent suitable for Xponders in a partially disaggregated scenario. The agent includes a gRPC-based protobuf-encoded telemetry streaming server. Similarly, [6] adopts an extended agent performing threshold-based telemetry streaming for network verification and data analytics. Work in [15] exploits a telemetry-based procedure to select operational modes (i.e., opaque attributes to identify transceiver transmission configuration set, including modulation, FEC, baud-rate and proprietary vendor-locked parameters) during elastic lightpath provisioning in a partially disaggregated scenario. Parallel OSNR and BER collectors of 2xPonder are deployed, equipped with online Grafana-based programmable graphic user interfaces, as shown in Fig.2.

\section{CONCLUSIONS}

The paper detailed the concept and the key aspects of telemetry services in SDN optical white box networks, including architectures, workflows, protocols, YANG models, along with the implementation state-of-the-art.

\section{REFERENCES}

[1] L. Velasco, A. C. Piat, O. Gonzlez, A. Lord, A. Napoli, P. Layec, D. Rafique, A. D'Errico, D. King, M. Ruiz, F. Cugini, and R. Casellas, "Monitoring and data analytics for optical networking: Benefits, architectures, and use cases," IEEE Network, vol. 33, no. 6, pp. 100-108, 2019.

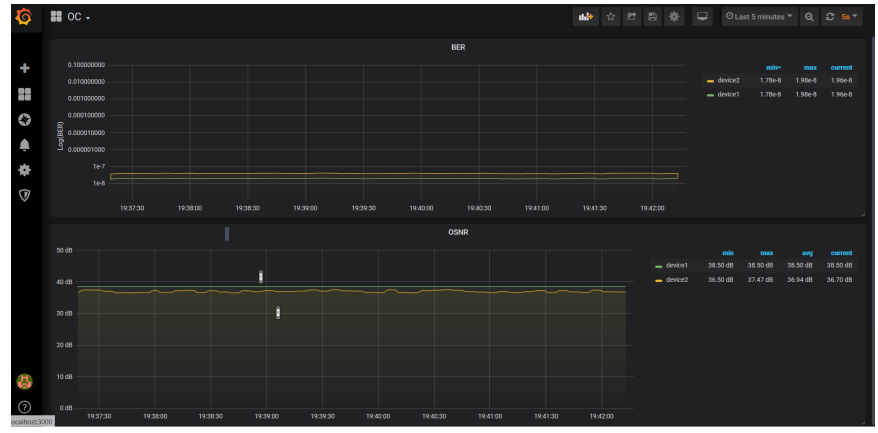

Fig. 2. Parallel telemetry of Multi-Xponder OSNR and BER collectors with Grafana-based GUI [15]

[2] B. Shariati, M. Ruiz, J. Comellas, and L. Velasco, "Learning from the optical spectrum: Failure detection and identification," Journal of Lightwave Technology, vol. 37, no. 2, pp. 433-440, Jan 2019.

[3] F. Paolucci, A. Sgambelluri, F. Cugini, and P. Castoldi, "Network telemetry streaming services in SDN-based disaggregated optical networks," Journal of Lightwave Technology, vol. 36, no. 15, pp. 3142-3149, 2018.

[4] B. Gangopadhyay, J. Pedro, J. Santos, N. Borges, P. Santos, H. Bock, N. Prasad, and R. Prasad, "Evaluating the cost benefit of real-time optical performance in dynamic optical restoration," in International Conference on the Design of Reliable Communication Networks (DRCN), 2020.

[5] L. Velasco, A. Sgambelluri, R. Casellas, L. Gifre, J. Izquierdo-Zaragoza, F. Fresi, F. Paolucci, R. Martínez, and E. Riccardi, "Building autonomic optical whitebox-based networks," Journal of Lightwave Technology, vol. 36, no. 15, pp. 3097-3104, Aug 2018

[6] A. Sadasivarao, S. Syed, D. Panda, P. Gomes, R. Rao, J. Buset, L. Paraschis, J. Brar, and K. Raj, "Demonstration of extensible thresholdbased streaming telemetry for open dwdm analytics and verification," in Optical Fiber Communication Conference. Optical Society of America, 2020, pp. M3Z-5.

[7] Y. Lee, R. Vilalta, R. Casellas, R. Martínez, and R. Muñoz, "Scalable telemetry and network autonomics in actn sdn controller hierarchy," in 2017 19th International Conference on Transparent Optical Networks (ICTON), July 2017, pp. 1-4.

[8] L. Velasco, L. Gifre, J. L. Izquierdo-Zaragoza, F. Paolucci, A. P. Vela, A. Sgambelluri, M. Ruiz, and F. Cugini, "An architecture to support autonomic slice networking," Journal of Lightwave Technology, vol. 36, no. 1, pp. 135-141, 2018.

[9] L. Gifre, J. Izquierdo-Zaragoza, M. Ruiz, and L. Velasco, "Autonomic disaggregated multilayer networking," IEEE/OSA Journal of Optical Communications and Networking, vol. 10, no. 5, pp. 482-492, 2018.

[10] S. Xu, Y. Hirota, M. Shiraiwa, M. Tornatore, S. Ferdousi, Y. Awaji, N. Wada, and B. Mukherjee, "Emergency opm recreation and telemetry for disaster recovery in optical networks," Journal of Lightwave Technology, pp. 1-1, 2020.

[11] T. Tanaka, S. Kuwabara, H. Nishizawa, T. Inui, S. Kobayashi, and A. Hirano, "Field demonstration of real-time optical network diagnosis using deep neural network and telemetry," in 2019 Optical Fiber Communications Conference and Exhibition (OFC), March 2019.

[12] K. Ishii, S. Yanagimachi, A. Tajima, and S. Namiki, "Submilisecond control/monitoring of disaggregated optical node through a direct memory access based architecture," in 2019 Optical Fiber Communications Conference and Exhibition (OFC), March 2019, pp. 1-3.

[13] J. Kundrat, O. Havlis, J. Radil, J. Jedlinsky, and J. Vojtech, "Opening up roadms: a filterless add/drop module for coherent-detection signals," IEEE/OSA Journal of Optical Communications and Networking, vol. 12, no. 6, pp. C41-C49, June 2020.

[14] A. Sgambelluri, A. Giorgetti, F. Paolucci, P. Castoldi, and F. Cugini, "Open source implementation of OpenConfig telemetry-enabled NETCONF agent," in 2019 21st International Conference on Transparent Optical Networks (ICTON), 2019, pp. 1-4.

[15] A. Sgambelluri, F. Paolucci, and F. Cugini, "Telemetry-driven validation of operation modes in OpenConfig disaggregated networks," in ECOC 2019; 45nd European Conference on Optical Communication, Sept. 2019, p. W3E3. 